\title{
Single Sampling Versus Multiple Testing Strategy to Assess Gut Microbiota Composition: Does It Matter?
}

\author{
T. G. J. de Meij ${ }^{1}$ - A. E. Budding ${ }^{2}$
}

Received: 2 February 2018 / Accepted: 26 March 2018 / Published online: 28 March 2018

(c) Springer Science+Business Media, LLC, part of Springer Nature 2018

With great interest we read the study by Su et al. concluding that microbiota analysis of faecal samples collected at 1 day could be used to describe microbiota composition. In contrast, since the alpha diversity of 5-day faecal samples was higher than that of 1-day faecal samples, they recommended the use of 5-day faecal samples to measure microbial diversity [1]. We agree with the authors that intraindividual microbial dynamics should be taken into account when assessing healthy individuals' gut microbiota signature, both in population-wide studies and in single individuals. We would, however, like to add some considerations concerning the authors' recommendation to apply a singlesampling strategy to describe microbiota composition. We recently performed a study on microbial composition and dynamics on faecal samples from 61 healthy children aged 2-18 years [2]. Samples were collected weekly, for 6 weeks, and a follow-up sample after 18 months. We observed that compositional stability through time varied per time point ( $p$ value $<0.0005$ ), even for a 1 -week interval. These stability patterns were phylum-specific $(p$ value $<0.0005)$, with the highest stability for Bacteroidetes, followed by Proteobacteria and Firmicutes/Actinobacteria. Overall compositional stability was on average $70 \%$ over a period of 18 months. Shannon diversity indices were stable over time. Possible explanation for the apparent discrepancy in outcome between the study of Su and our study could be differences in number of study participants ( 8 versus 61 , respectively). Furthermore, Su et al. described microbiota of samples collected at five consecutive days while we analysed samples weekly, for a total of 6 weeks. Also, differences in age (adults versus children) may have influenced outcome. However, children aged $>3$ years are considered to have a more or less adult microbiota composition. Based on our results we suggested that the observed intra-individual dynamics could have implications for strategies aiming at describing healthy individuals' gut microbiota signature. The frequently applied single-sampling (cross-sectional) strategy to analyse microbiota composition may not accurately reflect the compositional variability through time. We believe that calculation of the average composition measured at different time points could possibly provide a more integral view. Such an approach of multiple sampling is daily practice in the diagnostic work-up of, for example, parasitic infections, in which a triple faeces test is performed to increase test accuracy.

\section{Compliance with Ethical Standards}

Conflict of interest TGJ de Meij served in the advisory board of Danone. A.E. Budding has proprietary rights on the IS-pro platform technology and is co-founder of a spin-off company developing this technique into a clinical diagnostic product.

\section{References}

1. Su T, Liu R, Long Y, Quan S, Lai S, Wang L, Si J, Chen S (2018) 1-Day or 5-day fecal samples, which one is more beneficial to be used for DNA-based gut microbiota study. Curr Microbiol. https ://doi.org/10.1007/s00284-017-1378-8

2. de Meij TG, Budding AE, de Groot EF, Jansen FM, Frank Kneepkens CM, Benninga MA, Penders J, van Bodegraven AA, Savelkoul PH (2016) Composition and stability of intestinal microbiota of healthy children within a Dutch population. FASEB J 30(4):1512-1522. https://doi.org/10.1096/ff.15-278622

T. G. J. de Meij

t.demeij@vumc.nl

1 Department of Pediatric Gastroenterology, VU University Medical Centre, Amsterdam, The Netherlands

2 Department of Medical Microbiology and Infection Control, VU University Medical Centre, Amsterdam, The Netherlands 